\title{
Intelligent Oven in Smart Home Environment
}

\author{
Bojun Li, Piyanuch Hathaipontaluk, Suhuai Luo \\ School Of Design, Communication \& Information Technology \\ The University of Newcastle, Callaghan NSW 2308 \\ suhuai.luo@newcastle.edu.au
}

\begin{abstract}
Smart homes are gradually becoming one of the main applications in the high technology area. The intelligent appliance is a fundamental component of a smart home environment. People tend to invest in the kitchen appliances as they will be used throughout their lifetime, therefore many manufacturers focus on making these appliance more interesting. However, majority of manufacturers focus on how to make them cook faster. Very few manufacturers pay attention on the ability to cook healthier food. In this paper we introduce an innovative intelligent oven for the healthier food choice that is woven inside smart home environment. The intelligent oven is designed for manipulating recommended healthy recipe choices to suit each family member health concerns. Moreover, its ability to interact with other smart appliances such as smart fridge, mobile phone, and smart fire alarm are beneficial. We believe that the features mentioned above will make the intelligent oven an essential component in the smart home environment.
\end{abstract}

\section{Introduction}

Smart home is a living environment that consists of sensors, actuators, network and middle ware to provide appropriate service to the home's occupants automatically [2]. It has major usages for environmental, security, home entertainment, domestic appliances, information and communication and health $[1,3,5]$. About 20 home labs are set for researching on smart homes by research groups such as MIT, Siemens, Cisco, IBM, Xerox, Microsoft, etc. More than 30 appliances, at least 5 network protocols and over 3 artificial intelligence techniques have been used to conduct the research [4].

Smart appliances are fundamental components of a smart home environment. Embedded systems such as sensors, controllers, RFID, blue tooth devices and other wearable computers, along with the wireless mobile communication technology are the key factors $[5,6]$. Smart appliance research is conducted mainly in the area of security and hospitality to support individual elderly or disabled, or to provide comfort and support elderly live with their caretakers [7].
Example of the smart appliances for security purpose is the smart bed that detects a residence's unusual heart beat, or the smart floor equipped with weight sensor and software to detect unusual behaviour of residences [4]. These intelligent appliances are the main trend that will eventually replace traditional appliances. It is because they can notably reduce the amount of manual interactions necessitated by the inhabitants.

Kitchen is a prominent area in the house consisting of various intelligent appliances [15] such as refrigerator, cook top, dish washer, oven, and etc. Although ovens are among the most maturely developed products, majority of manufacturers focus on how to make them cook faster by combining main features of different existing models such as steam oven, convection oven, and microwave oven. Very few of the industrial developers pay attention to producing intelligent ovens with the ability to cook healthier food although oven-cooking appeared to have less fat and more protein from the nutritional perspective [16].

Research has shown that there are many occurance of malnutrition and reducing in nutritional value of food as a result of using wrong cooking methods [12]. The using of thermometer to check the doneness of the cooking meat products is pushed to be national campaigns in USA in 1998 and 2005 [13]. As a result, controlling of cooking temperature is an important function in assuring that meat is well cooked to kill harmful bacteria [12]. However, using high temperature potentially destroy certain vitamins and minerals in food [14].

Consequently, we have developed database and interface of an intelligent oven system to demonstrate it's capability of suggesting healthy food choices for each inhabitant. Along with the ability to interact with other smart devices in the smart home environments such as smart fridge and smart fire alarm. It can be controllable by the user mobile phone. The detailed functions will be discussed in this paper.

In the following, we first describe our novel intelligent oven in section 2, then present the system design in section 3, and finally make conclusions in section 4 .

\section{Intelligent oven with healthy diet feature}

The ovens produced with advanced technology by famous corporations [8-11] are concerned on the 
multiply functions as well as multimedia and network application. On the other hand, these main manufactures pay more and more attention to designing and building advanced features to make the oven more likely to be a super machine which can provide almost all the functions for human needs. However, they neglect the fact that health issues about cooking and nutrient intake are paid more attention by an increasing number of people in the world. In this section, we present our approach in this area.

\subsection{Perspective of the project}

The main perspective of the design is to develop a database that contains standard nutritional information and integrate it to the intelligent oven's central processor, and an interface that allows user to easily operate the oven and manipulate nutritional reports. Each user will record his or her personal information such as date of birth, height, weight, diseases, etc. The pre-programmed recipes contain ingredients, instruction and nutrition facts information. When a user presses start cooking button, the user is requested to specify who will have the recipe including guests who want to share the meal. Then the oven records each member's recipes cooked including nutrients intake which is used to generate nutritional report. The oven can display the standard nutrient values or the estimated energy requirement according to the user gender, age, weight and height. An exercise program can be produced according to the recipe selected or the energy level enters by the user.

\subsection{The unique features}

Nutritional function of the intelligent oven provides users a guideline of the healthy recipe choices to suit each member preferences in addition to recording only the favourite recipes. Personal data of each member are recorded and every recipe cooked by each member is stored each time the oven is used. Then a nutritional report is generated by comparing the nutrient intake from a user with the standard nutrient values or the estimated energy requirement.

An intelligent oven interacting with other smart devices including smart fridge and smart fire alarm system in a smart home is another main benefit. The oven can retrieve data from the smart fridge to generate recipe choices for user according to the ingredients left in the fridge. The additional function for recording family event allows user to plan for grocery shopping and cooking for the upcoming party. Moreover, it can monitor internal cooking temperature using multiple sensors to detect temperature from multi-spot of the cooking item. When the temperature reaches the safety point, it will trigger the smart fire alarm system to operate.

\subsection{Functionality of our intelligent oven}

There are nine major functions in our intelligent oven, including:

- Recording members' personal and health information including name, age, gender, height, weight, disease, vitamin deficiency, and food allergic.

- Interacting with the smart fridge to retrieve ingredients information to generate shopping list and recipes according to ingredients available

- Recording family events and expected recipe to allow user to prepare grocery shopping and cooking plan

- Recipe manipulating according to user's health concern and preference. The function includes creating favourite recipes, starting cooking the selected recipe, monitoring internal temperature, and activating fire alarm

- Generating exercise program according to recipe selected or the energy amount user entered

- Recording cooking history every time the oven is used

- Monitoring nutrients intake for each user

- Generating nutritional report and analysing nutritional data to produce a nutritional guideline for users

- Mobile phone remote controlling to start and stop cooking food

\section{System design}

\subsection{System architectures}

For the smart oven system, we use Microsoft SQL Server 2008 to build the database and use Microsoft Visual Studio 2008 to create the user interface.

The system is two-tier architecture. User interacts with the interface of the smart oven. The database is connected to the VB.NET codes through applying SQL Server classes. In the VB code section, most of the functions are manipulated through using stored procedures.

\subsection{Database design}

There are 16 database tables, including:

FamilyMember: contains user's all personal information

Nutrient_Taken_Recommendation: contains Member_id, Nutrient_id, limitation_weight. Exercise_Program: includes EP_id, Program_Name and Energy_Burned, representing each program the energy burned during certain time.

Family_Event: includes Event_id, Description and Event Date.

Coooking_Planning: represents the cooking plan details

Recipe: includes Recipe_id, Recipe_Name, 
Cooking_Method etc., reprensenting all the recipe information. All users can choose recipe easily. Recipe_Cooked: contains RC_id, Member_id, Datetime_Cooked and People_Served. This is a middle table between recipe and recipe table.

Recipe_Nutrient: contains RN_id, Recipe_id, Nutrient_id and Nutrient_weight, representing Recipe's nutrient information.

Recipe_Ingredient: includes Recipe_id, ingredient_id, Ingredient_Weight and Ingredient_Units. This is the middle table connecting the Recipe table and Ingredient table. It represents how many ingredients each recipe uses

Ingredient: includes Ingredient_id, Ingredient_Name and Allergen. It records all detailed Ingredient information

Nutrient_Facts: includes Nutrient_id, Nutrient_Name and Unit. It records all the Nutrient information.

Sensor: includes Sensor_Name, Functions, Numbers, Current_temp. It monitors the temperature of internal food.

Fridge_Ingredient: contains Name, weight, unit, purchaseDate etc., which is retrieved from Smart Fridge database.

Estimated_Energy_Requirement: records all the information of different people's daily enery intake standard

Nutrient_Reference_Values: records all the information of different gender group daily Nutrient intake standard like VitamineA, VitamineC, Protein etc.

Member_Favorite: connects tables called

FamilyMember and Recipe by using Member_id and Recipe_id.

The relationships between those tables are shown in the ER diagram as Fig. 1.

\subsection{Interface design}

This section we present the main function panel and four of the nine sub-functions that users use to interact with the system.

- The intelligent oven control panel has nine main function pages for user to interact with the system (Fig. 2).

- Recipes panel contains sub-functions such as oven cooking control panel which monitors cooking temperature and activates the fire alarm system, search recipe by condition, manage user's favourite recipe, add new recipe to the database, and check exercise program for the selected recipe

- Member function panel allows user to add, modify and delete user information to the oven. User can view all users' information using detail button

- Refrigerator panel enables user to check the available ingredient in the smart fridge and produce the shopping list

Monitor Nutrients panel displays all the nutrients user had compare with the standard nutrient values

\section{Conclusion and future work}

We have introduced a novel smart oven with nutrient intake and food healthy monitor capability. The proposed system has shown its great characteristics in maintaining better nutrient intake and health diet. There are still some places that can be improved for the system in the future. Possible extensions include: Generate daily recipes or weekly recipe plan according to the previous nutrient intake unhealthily; Automatic add sauce and seasoning after put food in the oven.; Connecting to smart fridge, and show its interface on oven if needed; Control the microwave system through smart oven interface; Online recipe real time update, the recipe in the intelligent oven should be updated through connecting internet.

\section{Reference}

[1] Housing Learning \& Improvement Network factsheet. Intertek Research \& Testing Center for the Department of Trade and Industry, DTI Smart Homes Project. Sep, 2003

[2] Choi J., Shin D., and Shin D.: Research on Design and Implementation of the Artificial Intelligent Agent for Smart Home Based on Support Vector Machine. ICNC, (2005) 1185-1188

[3] Marie Chan, Daniel Esteve, Christophe Escriba, Eric Campo.: A review of smart homes- Present state and future challenges. Computer Methods and Programs in Biomedicine 91. (2008), pp. 55-81

[4] Jiang L., Liu D.Y., and Yang B.: Smart Home Research. Proceedings of the $3^{\text {rd }}$ International Conference on Machine Learning and Cybernetics. IEEE, (2004) 659-663

[5] Seung-Ho Baeg, Jae-Han Park, Jaehan Koh, KyungWook Park, Moon-Hong Baeg.: Building a Smart Home Environment for Service Robots Based on RFID and Sensor Networks. Int. Conf. on Control, Automation and System 2007. Oct, 2007

[6] Sajal K. D., Diane J. C.: Designing and Modelling Smart Environments (Invited Paper). International Symposium on World of Wireless, Mobile and Multimedia Networks, (2006) [7] Bates D.W., and Gawande A.A.: Patient Safety: Improving Safety with Information Technology. The New England Journal of Medicine, 348:25, (2003) 2526-2534

[8] http://www.appliancist.com/builtin ovens/ Samsung-dualcooking-twin-convection- oven.html (accessed on March 8, 2009)

[9] http://www. appliancist.com/builtin ovens/ Electroluxicon-professional-series-hi- speed- oven.html (accessed on March 8, 2009)

[10] http://www.miele.com/usa/cooking/ovens/ product.asp (accessed on March 20, 2009)

[11] Garate A, Lucas I, Herrasti N, Lopez A (2005), Ambient 
Intelligence as paradigm of a full Automation Process at Home in a real application, Proceedings IEEE International Symposium on Computational Intelligence in Robotics and Automation.

[12] Zhuang H. and Savage E.M.: Sensory and Food Quality: Validation of a Combi Oven Cooking Method for Preparation of Chicken Breast Meat for Quality Assessment. Journal of food science, Vol.73, Nr.8, (2008) p424-430.

[13] Mccurdy S. M., Takeuchi M. T., et al:Food Safety education initiative to increase consumer use of food thermometers in the United States. British Food Journal Vol 108 No.9, (2006) pp. 775-794

[14] Joonseok Park, Mikyeyong Moon, Seongjin Hwang,
Keunhyuk Yeom.: Development of Simulation System for Validating Contextual Rule in Smart Home. Feb. 12-14, 2007. ICACT2007

[15] Luo, S., Xia, H., Gao, Y., Jin, J. S., Athauda, R., Smart Fridges with Multimedia Capability for Better Nutrition and Health, The 2008 International Symposium on Ubiquitous Multimedia Computing, Hobart, Australia, October 13 - 15, pp. 39-44, 2008.

[16] Marzena Danowska-Oziewicz, Miroslawa KarpinskaTymoszczyk and Jerzy Borowski.: The effect of cooking in a steam-convection oven on the quality of selected dished. Journal of Foodservice, 18. pp. 187-197 Blackwell Publishing.

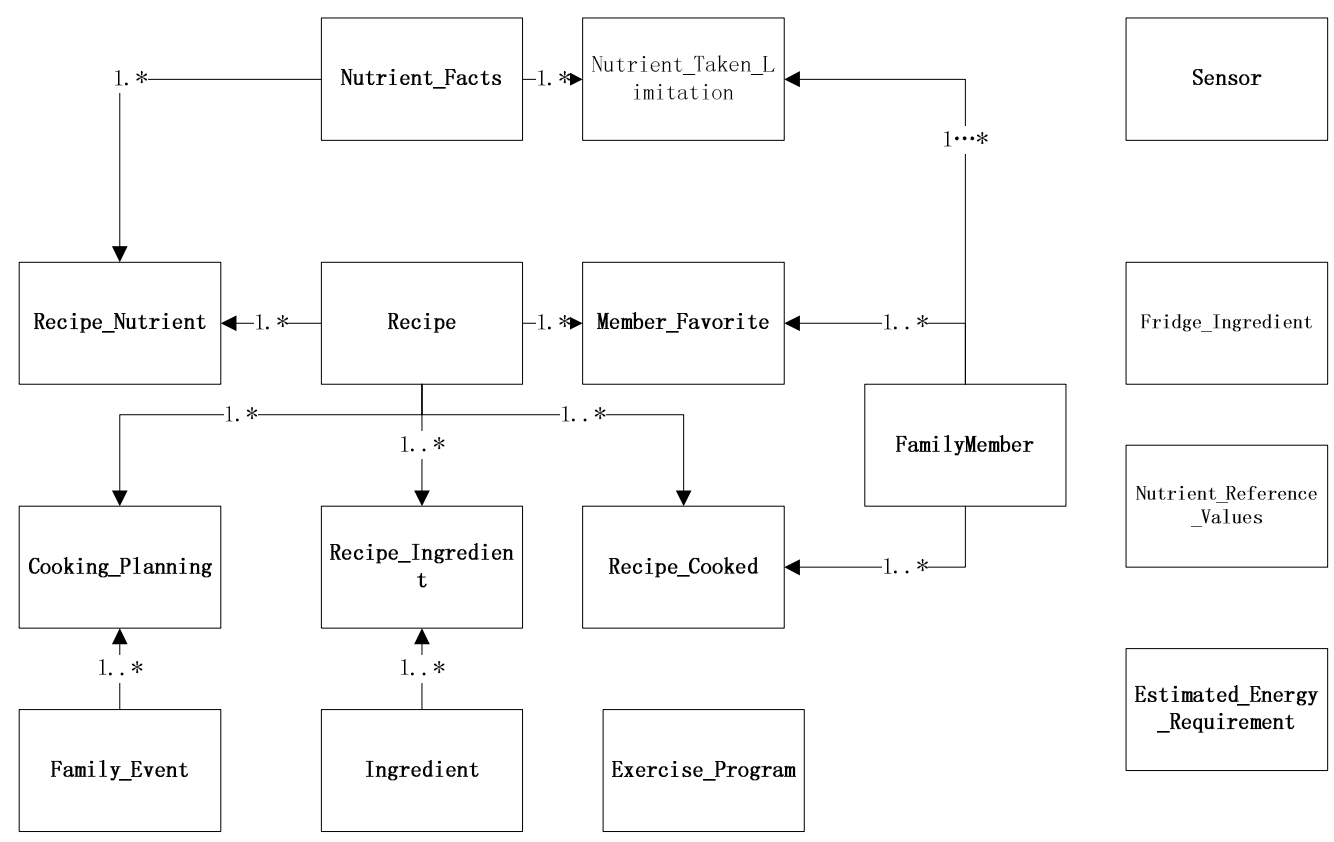

Fig. 1. Intelligent Oven E-R Diagram. Where square block indicates entity; connecting line indicates relationships between entities; 1 indicates single relationship; *indicate multiple relationship.

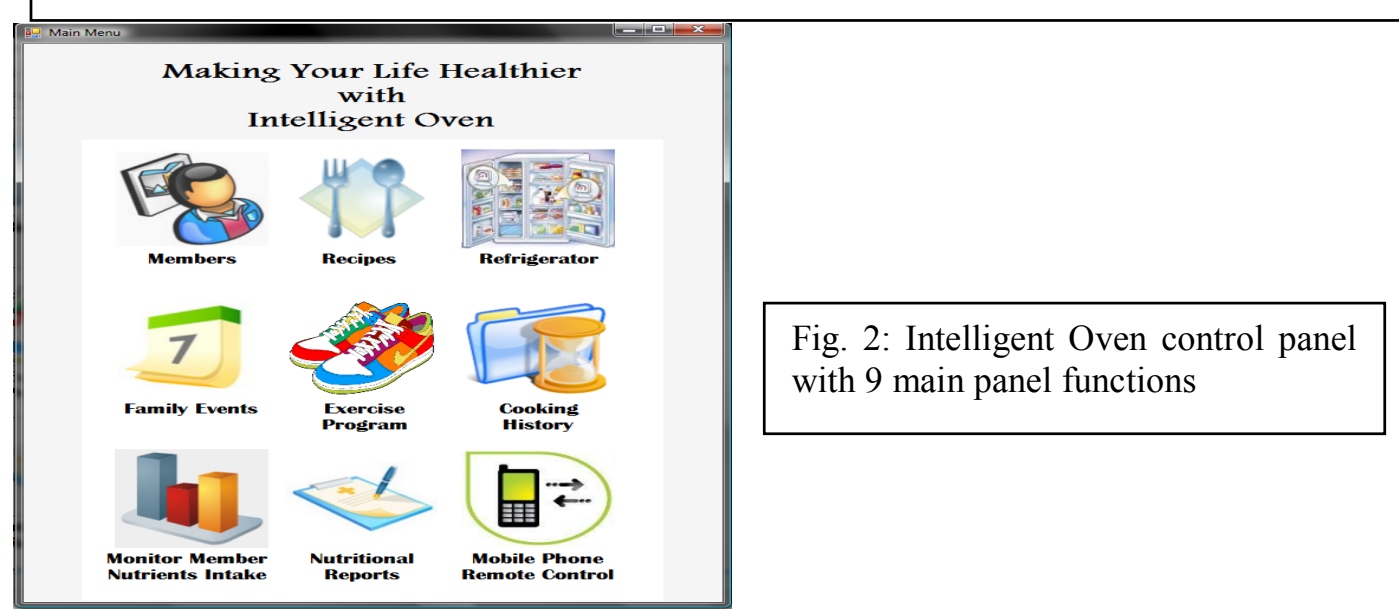

